\title{
Antitumor Agents. 269. Non-Aromatic Ring-A Neo-tanshinlactone Analog, TNO, as a New Class of Potent Antitumor Agents
}

\author{
Yizhou Dong ${ }^{\mathrm{a}}$, Qian Shi ${ }^{\mathrm{a}}$, Kyoko Nakagawa-Goto ${ }^{\mathrm{a}}$, Pei-Chi Wu ${ }^{\mathrm{a}}$, Kenneth F. Bastow ${ }^{\mathrm{b}}$, Susan \\ L. Morris-Natschke ${ }^{a}$, and Kuo-Hsiung Lee ${ }^{a,{ }^{*}}$ \\ aNatural Products Research Laboratories, Eshelman School of Pharmacy, University of North \\ Carolina, Chapel Hill, North Carolina 27599 \\ bDivision of Medicinal Chemistry and Natural Products, Eshelman School of Pharmacy, University \\ of North Carolina, Chapel Hill, North Carolina 27599
}

\begin{abstract}
Tetrahydroneotanshinlactone (TNT) and tetrahydronaphthalene-1-ol (TNO) derivatives were designed, synthesized, and evaluated for cytotoxic activity. The TNO derivatives were found to be a promising novel class of in vitro antitumor agents. The cyclohexene ring-A could dramatically affect the antitumor activity and selectivity. Compound $\mathbf{2 0}$ showed the highest potency with $\mathrm{ED}_{50}$ values of 0.7 and $1.7 \mu \mathrm{M}$ against SK-BR-3 and ZR-75-1 breast cancer cell lines, respectively.
\end{abstract}

\section{Keywords}

Tetrahydronaphthalene-1-ol (TNO) analogs; Tetrahydroneotanshinlactone (TNT) analogs; Cytotoxicity; Breast cancer

Cancer is the second leading cause of death in the United States, accounting for one-quarter of all deaths. ${ }^{1}$ Cancer incidence and resultant mortality have increased by approximately $22 \%$ since $1990,{ }^{2}$ and $1,400,000$ new cancer cases will be diagnosed in 2009, according to estimates by the American Cancer Society. ${ }^{3}$ Natural products and natural product-derived compounds have provided more than two-thirds of the clinically used anticancer agents, and thus, are important sources for drug discovery. ${ }^{4,5}$ Plant-based drug discovery has resulted particularly in the development of new anticancer agents to by-pass multidrug resistance and overcome side effects of current therapeutic medicines. ${ }^{6,7}$ The above facts prompted us to focus on natural products and their analogs in our anticancer drug program.

Research on tanshinones (isolated from the Salvia genus) began in the early 1930s. ${ }^{8}$ Tanshinone I (1) and tanshinone IIA (2) differ structurally in the ring-A system: the former has an aromatic ring, while the latter has a non-aromatic ring (Figure 1). Compounds $\mathbf{1}$ and $\mathbf{2}$ have been studied extensively for their antitumor effects, and display different activities and selectivities. ${ }^{8}$ Recent studies indicated that $\mathbf{1}$ reduced metastasis and tumorigenesis by inhibition of IL- $8,{ }^{9}$ while $\mathbf{2}$ induced cell differentiation and apoptosis. ${ }^{10}$ Neo-tanshinlactone

(C) 2009 Elsevier Ltd. All rights reserved.

*Corresponding author. Tel: 919-962-0066; fax: 919-966-3893; khlee@unc.edu.

Publisher's Disclaimer: This is a PDF file of an unedited manuscript that has been accepted for publication. As a service to our customers we are providing this early version of the manuscript. The manuscript will undergo copyediting, typesetting, and review of the resulting proof before it is published in its final citable form. Please note that during the production process errors may be discovered which could affect the content, and all legal disclaimers that apply to the journal pertain. 
(3) (Figure 1), reported by our group previously, ${ }^{11}$ showed significant and selective in vitro anti-breast cancer activity. We further studied how the individual rings in $\mathbf{3}$ influence the in vitro activity, and the results led to the discovery of a novel class of potential anti-breast cancer agents, 2-(furan-2-yl)naphthalen-1-ol derivatives, such as analog 4. However, it remained unclear how ring A affects the activity and selectivity of 3-and 4-analogs. To answer this question, we designed derivatives with two new scaffolds, tetrahydroneotanshinlactone $(\mathbf{5}$, TNT) and tetrahydronaphthalene-1-ol (6, TNO). Like 2, both TNT and TNO derivatives have a non-aromatic ring-A. Different ring sizes, including five- and six-membered rings, were studied and 14 new analogs were designed. This paper reports the synthesis and biological evaluation of 5- and 6-analogs.

As shown in Scheme 1, compound $\mathbf{8}$ was synthesized by Negishi cross-coupling reaction of compound 7 with 4-methylpent-3-enyl zinc(II) bromide in $96 \%$ yield. ${ }^{12,}{ }^{13}$ Treatment of 8 with $\mathrm{AlCl}_{3}$ followed by demethylation gave 9 in $84 \%$ yield. ${ }^{13}$ Compounds $\mathbf{1 0}$ and $\mathbf{1 1}$, with five- and six-membered rings, respectively, are commercially available. Compounds 9-11 underwent the previously reported two-step ring closure reactions to afford furochromenones 15-17, which were hydrolyzed by using sodium hydroxide to give ring-opened compounds 18-20. Compound 20, with gem-dimethyl substitution on ring-A, showed significant cytotoxic activity (Table 1), and was chosen for further modification to study the functions of the hydroxyl and carboxylic acid groups (21-28). The selective methylation of the hydroxy group on $\mathbf{2 0}$ was achieved by the addition of MeI and 18-crown-6 ether to the crude hydrolysis mixture of $\mathbf{1 7}$ without work-up. ${ }^{14}$ The resulting carboxylic acid $\mathbf{2 1}$ was converted to methyl ester $\mathbf{2 2}$ with thionyl chloride and $\mathrm{MeOH}$ at room temperature. Meanwhile, the reduction of $\mathbf{2 0}$ with lithium aluminum hydride afforded diol $\mathbf{2 3}$, which was treated with iodomethane and iodoethane in the presence of $\mathrm{Cs}_{2} \mathrm{CO}_{3}$ to generate ethers 24 and 25 , respectively. The remaining primary alcohol of $\mathbf{2 4}$ was alkylated with iodomethane and iodoethane in the presence of $\mathrm{NaH}$ to obtain $\mathbf{2 6}$ and $\mathbf{2 7}$, respectively. Acetate $\mathbf{2 8}$ was obtained by acetylation of $\mathbf{2 4}$ with $\mathrm{Ac}_{2} \mathrm{O}$.

The newly synthesized analogs $\mathbf{1 5}-\mathbf{2 8}$ were tested for in vitro cytotoxic activity against a panel of human tumor cell lines according to previously published methods. ${ }^{15}$ Cell lines include: SK-BR-3 (estrogen receptor negative, HER2 over-expressing breast cancer), ZR-75-1 (estrogen receptor positive breast cancer), MDA-MB-231 (estrogen receptor negative breast cancer), A549 (non small cell lung cancer), DU145 (prostate cancer cell line), KB (nasopharyngeal carcinoma), and KB-vin (vincristine-resistant MDR KB subline).

Among the three tetrahydroneotanshinlactone (TNT) derivatives, $\mathbf{1 5}$ showed no activity against any tumor cell line tested, which suggested that the five-membered ring A was not favored. Compound 16 was three- to seven-fold more potent than 17 against SK-BR-3, ZR-75-1, A549, and KB-vin cell lines. However, while $\mathbf{1 6}$ was less potent compared with $\mathbf{3}$ against SK-BR-3 and ZR-75-1 breast cancer cell lines, it also showed a broader antitumor spectrum, with greatly enhanced potency against A549 and KB-vin. The results suggested that ring-A could affect the potency and tumor-tissue type selectivity dramatically.

Among tetrahydronaphthalene-1-ol (TNO) derivatives, compounds 18 and 19 displayed only marginal antitumor activity, while $\mathbf{2 0}$ showed potent and broad antitumor activity against all tumor cell lines tested ( $\mathrm{ED}_{50} 0.7 \mu \mathrm{M}$ against SK-BR-3; $1.7 \mu \mathrm{M}$ against $\left.\mathrm{ZR}-75-1\right)$. Thus, a nonaromatic six-membered ring-A with gem-dimethyl substitution was favored for cytotoxic activity, in comparison with unsubstituted five- and six-membered rings. As to the tumor-tissue type selectivity, 20 was significantly active against all tumor cell lines tested, except MDAMB-231, while 3 and $\mathbf{4}$ were active against only two of the breast cancer cell lines. In contrast to 20, compounds $\mathbf{3}$ and $\mathbf{4}$ lack the gem-dimethyls, and are essentially planar. These results demonstrated that, by changing the molecular conformation and orientation, introduction of a non-aromatic ring-A could greatly influence the antitumor activity against all cell types. In our 
prior SAR studies of neo-tanshinlactone (3) and the ring-opened analog 4 , the presence of two functional groups from the opened lactone ring- $\mathrm{C}$ was critical for antitumor activity, which encouraged us to study comparable derivatives of $\mathbf{2 0}$ with ether and ester groups of various sizes. As seen in Table 1, 21-28 showed only moderate to marginal activity against all tumor cell lines tested, but interestingly, still displayed low sensitivity against MDA-MB-231 compared with other tumor cell lines. For example, $\mathbf{2 5}$ and $\mathbf{2 8}$ showed four-fold higher potency against SK-BR-3 than MDA-MB-231. In summary, the current SAR study indicated that the optimal substituents on the phenyl and furanyl rings are hydroxy and carboxylic acid groups. The preliminary results indicated that the identities of the ring A, hydroxy, and carboxylic acid groups are important to antitumor activity and selectivity. More analogs will be synthesized and evaluated to establish detailed structure-activity relationships (SAR) of this new series of compounds.

In conclusion, tetrahydroneotanshinlactone (TNT) and tetrahydronaphthalene (TNO) derivatives were prepared in order to investigate the effect of the non-aromatic ring-A on in vitro antitumor activity. The results indicated that a non-aromatic ring-A could dramatically affect both activity and tumor cell line selectivity, particularly the non-breast cell lines that were studied. Based on this study, a novel class of antitumor agents, TNO derivatives, was discovered and developed. Compound $\mathbf{2 0}$ was the most potent analog with an $\mathrm{ED}_{50}$ value of $0.7 \mu \mathrm{M}$ against the SK-BR-3 cell line, and showed broader antitumor activity compared with 3 and 4. Further SAR and mechanism of action studies are ongoing and progress will be reported in due course. In summary, $\mathbf{2 0}$ is a promising new lead compound with a novel skeleton for further development toward a new potential clinical trials candidate.

\section{Acknowledgments}

This work was supported by NIH grant CA-17625 from the National Cancer Institute, awarded to K.H. Lee.

\section{References and notes}

1. Balunas MJ, Kinghorn AD. Life Sci 2005;78:431. [PubMed: 16198377]

2. Parkin DM. Lancet Oncol 2001;2:533. [PubMed: 11905707]

3. Cancer Facts \& Figures. American Cancer Society. 2009

4. Tan G, Gyllenhaal C, Soejarto DD. Curr. Drug Targets 2006;7:265. [PubMed: 16515527]

5. Paterson I, Anderson Edward A. Science 2005;310:451. [PubMed: 16239465]

6. Rishton GM. Am. J. Cardiol 2008;101:43D-49D. [PubMed: 18243858]

7. Saklani A, Kutty SK. Drug Discov. Today 2008;13:161. [PubMed: 18275914]

8. Wang X, Morris-Natschke SL, Lee KH. Med. Res. Rev 2007;27:133. [PubMed: 16888751]

9. Lee C-Y, Sher H-F, Chen H-W, Liu C-C, Chen C-H, Lin C-S, Yang P-C, Tsay H-S, Chen JJW. Mol. Cancer Ther 2008;7:3527. [PubMed: 19001436]

10. Sung HJ, Choi SM, Yoon Y, An KS. Exp. Mol. Med 1999;31:174. [PubMed: 10630370]

11. Wang X, Bastow KF, Sun CM, Lin YL, Yu HJ, Don MJ, Wu TS, Nakamura S, Lee KH. J. Med. Chem 2004;47:5816. [PubMed: 15509181]

12. Fischer C, Fu GC. J. Am. Chem. Soc 2005;127:4594. [PubMed: 15796523]

13. Danheiser RL, Casebier DS, Firooznia F. J. Org. Chem 1995;60:8341.

14. Glover SA, Golding SL, Goosen A, McCleland CW. J. Chem. Soc., Perkin Trans. 1 1981:842.

15. Wang X, Nakagawa-Goto K, Bastow KF, Don MJ, Lin YL, Wu TS, Lee KH. J. Med. Chem 2006;49:5631. [PubMed: 16942038]

16. Spectroscopic data. 1-Methyl-7,8-dihydrocyclopenta[h]furo[3,2-c] chromen-10(6H) - one $(\mathbf{1 5}):{ }^{1} \mathrm{H}$ NMR (300 MHz, $\left.\mathrm{CDCl}_{3}, \mathrm{ppm}\right): \delta 2.19\left(\mathrm{p}, J=7.5 \mathrm{~Hz}, 2 \mathrm{H}, \mathrm{CH}_{2} \mathrm{CH}_{2} \mathrm{CH}_{2}\right), 2.36(\mathrm{~d}, J=1.5 \mathrm{~Hz}, 3 \mathrm{H}$, $\left.\mathrm{CH}_{3}\right), 3.04$ (t, $\left.J=7.5 \mathrm{~Hz}, 2 \mathrm{H}, \mathrm{CH}_{2} \mathrm{CH}_{2} \mathrm{CH}_{2}\right), 3.14\left(\mathrm{t}, J=7.5 \mathrm{~Hz}, 2 \mathrm{H}, \mathrm{CH}_{2} \mathrm{CH}_{2} \mathrm{CH}_{2}\right), 7.19$ (d, $J=$ $7.8 \mathrm{~Hz}, 1 \mathrm{H}$, aromatic), 7.36 (q, $J=1.2 \mathrm{~Hz}, 1 \mathrm{H}, \mathrm{OCH}), 7.63$ (d, $J=8.1 \mathrm{~Hz}, 1 \mathrm{H}$, aromatic); HRMS 
Calcd for $\mathrm{C}_{15} \mathrm{H}_{13} \mathrm{O}_{3}\left(\mathrm{M}+\mathrm{H}^{+}\right)$: 241.0859, found: 241.0858. 1-Methyl-8,9-dihydro-6H-benzo[ $h$ ]furo [3,2-c]chromen-11(7H)-one (16): ${ }^{1} \mathrm{H}$ NMR (300 MHz, $\left.\mathrm{CDCl}_{3}, \mathrm{ppm}\right): \delta 1.80-1.86(\mathrm{~m}, 4 \mathrm{H}$, $\left.\mathrm{CH}_{2} \mathrm{CH}_{2} \mathrm{CH}_{2} \mathrm{CH}_{2}\right), 2.35\left(\mathrm{~d}, \mathrm{~J}=1.2 \mathrm{~Hz}, 3 \mathrm{H}, \mathrm{CH}_{3}\right), 2.84\left(\mathrm{t}, \mathrm{J}=5.7 \mathrm{~Hz}, 2 \mathrm{H}, \mathrm{CH}_{2} \mathrm{CH}_{2} \mathrm{CH}_{2} \mathrm{CH}_{2}\right), 2.94$ (t, $\left.J=6.0 \mathrm{~Hz}, 2 \mathrm{H}, \mathrm{CH}_{2} \mathrm{CH}_{2} \mathrm{CH}_{2} \mathrm{CH}_{2}\right), 7.01$ (d, $J=8.4 \mathrm{~Hz}, 1 \mathrm{H}$, aromatic), 7.34 (d, $J=0.9 \mathrm{~Hz}, 1 \mathrm{H}$, $\mathrm{OCH}), 7.51\left(\mathrm{~d}, J=8.1 \mathrm{~Hz}, 1 \mathrm{H}\right.$, aromatic); HRMS Calcd for $\mathrm{C}_{16} \mathrm{H}_{15} \mathrm{O}_{3}\left(\mathrm{M}+\mathrm{H}^{+}\right): 255.1016$, found: 255.1012. 1,6,6-Trimethyl-8,9-dihydro- $6 H$-benzo[ $h]$ furo[3,2-c]chromen-11(7H)-one (17). 38\% yield; mp 101-103 ${ }^{\circ} \mathrm{C}$; ${ }^{1} \mathrm{H}$ NMR (300 MHz, $\mathrm{CDCl}_{3}$, ppm): $\delta 1.33\left(\mathrm{~s}, 6 \mathrm{H}, \mathrm{C}\left(\mathrm{CH}_{3}\right)_{2}\right), 1.67-1.71(\mathrm{~m}$, $\left.2 \mathrm{H}, \mathrm{CCH}_{2} \mathrm{CH}_{2} \mathrm{CH}_{2}\right), 1.84-1.88\left(\mathrm{~m}, 2 \mathrm{H}, \mathrm{CCH}_{2} \mathrm{CH}_{2} \mathrm{CH}_{2}\right), 2.35$ (d, $\left.J=1.2 \mathrm{~Hz}, 3 \mathrm{H}, \mathrm{CH}_{3}\right), 2.97(\mathrm{t}, J=$ $6.3 \mathrm{~Hz}, 2 \mathrm{H}, \mathrm{CCH}_{2} \mathrm{CH}_{2} \mathrm{CH}_{2}$ ), 7.32 (d, $J=8.4 \mathrm{~Hz}, 1 \mathrm{H}$, aromatic), 7.35 (q, $\left.J=1.2 \mathrm{~Hz}, 1 \mathrm{H}, \mathrm{OCH}\right), 7.61$ (d, $J=8.7 \mathrm{~Hz}, 1 \mathrm{H}$, aromatic); HRMS Calcd for $\mathrm{C}_{18} \mathrm{H}_{19} \mathrm{O}_{3}\left(\mathrm{M}+\mathrm{H}^{+}\right)$: 283.1329, found: 283.1315. 2(4-Hydroxy-2,3-dihydro-1 $H$-inden-5-yl)-4-methylfuran-3-carboxylic acid (18): ${ }^{1} \mathrm{H} N \mathrm{MR}(300 \mathrm{MHz}$, $\mathrm{CD}_{3} \mathrm{OD}, \mathrm{ppm}$ ): $\delta 2.06$ (p, $J=7.5 \mathrm{~Hz}, 2 \mathrm{H}, \mathrm{CH}_{2} \mathrm{CH}_{2} \mathrm{CH}_{2}$ ), $2.20\left(\mathrm{~d}, J=0.9 \mathrm{~Hz}, 3 \mathrm{H}, \mathrm{CH}_{3}\right), 2.89$ (q, $J$ $\left.=7.5 \mathrm{~Hz}, 4 \mathrm{H}, \mathrm{CH}_{2} \mathrm{CH}_{2} \mathrm{CH}_{2}\right), 4.94(\mathrm{~s}, 1 \mathrm{H}, \mathrm{OH}), 6.83(\mathrm{~d}, J=7.8 \mathrm{~Hz}, 1 \mathrm{H}$, aromatic), $7.14(\mathrm{~d}, J=7.8$ $\mathrm{Hz}, 1 \mathrm{H}$, aromatic), 7.30 (d, $J=0.9 \mathrm{~Hz}, 1 \mathrm{H}, \mathrm{OCH}$ ); MS: $m / z, 257\left(\mathrm{M}-\mathrm{H}^{+}\right)$. 2-(1-Hydroxy-5,6,7,8tetrahydronaphthalen-2-yl)-4-methylfuran-3-carboxylic acid (19): ${ }^{1} \mathrm{H}$ NMR (300 MHz, $\mathrm{CD}_{3} \mathrm{COCD}_{3}$, ppm): $\delta$ 1.75-1.77 (m, 4H, $\left.\mathrm{CH}_{2}\right), 2.20$ (d, $\left.J=1.2 \mathrm{~Hz}, 3 \mathrm{H}, \mathrm{CH}_{3}\right), 2.69-2.75(\mathrm{~m}, 4 \mathrm{H}$, $\left.\mathrm{CH}_{2}\right) 6.67(\mathrm{~d}, J=8.4 \mathrm{~Hz}, 1 \mathrm{H}$, aromatic), 7.10 (d, $J=8.4 \mathrm{~Hz}, 1 \mathrm{H}$, aromatic), 7.43 (s, $1 \mathrm{H}, \mathrm{OCH})$; HRMS Calcd for $\mathrm{C}_{16} \mathrm{H}_{15} \mathrm{O}_{4}\left(\mathrm{M}-\mathrm{H}^{+}\right)$: 271.0970, found: 271.0971. 2-(1-Hydroxy-5,5dimethyl-5,6,7,8-tetrahydronaphthalen-2-yl)-4-methylfuran-3-carboxylic acid (20): ${ }^{1} \mathrm{H}$ NMR (300 $\left.\mathrm{MHz}, \mathrm{CD}_{3} \mathrm{OD}, \mathrm{ppm}\right): \delta 1.28\left(\mathrm{~s}, 6 \mathrm{H}, \mathrm{C}\left(\mathrm{CH}_{3}\right)_{2}\right), 1.62-1.66\left(\mathrm{~m}, 2 \mathrm{H}, \mathrm{CH}_{2}\right), 1.78-1.82\left(\mathrm{~m}, 2 \mathrm{H}, \mathrm{CH}_{2}\right)$, $2.21\left(\mathrm{~d}, J=1.5 \mathrm{~Hz}, 3 \mathrm{H}, \mathrm{CH}_{3}\right), 2.70\left(\mathrm{t}, J=6.3 \mathrm{~Hz}, 2 \mathrm{H}, \mathrm{CH}_{2}\right), 6.96(\mathrm{~d}, J=8.4 \mathrm{~Hz}, 1 \mathrm{H}$, aromatic), 7.13 (d, $J=8.4 \mathrm{~Hz}, 1 \mathrm{H}$, aromatic), $7.33(\mathrm{~d}, J=1.2 \mathrm{~Hz}, 1 \mathrm{H}, \mathrm{OCH})$; HRMS Calcd for $\mathrm{C}_{18} \mathrm{H}_{19} \mathrm{O}_{4}(\mathrm{M}$ $-\mathrm{H}^{+}$): 301.1434, found: 301.1425. 2-(1-Methoxy-5,5-dimethyl-5,6,7,8-tetrahydronaphthalen-2yl)-4-methylfuran-3-carboxylic acid (21): ${ }^{1} \mathrm{H}$ NMR (300 MHz, $\left.\mathrm{CDCl}_{3}, \mathrm{ppm}\right): \delta 1.30(\mathrm{~s}, 6 \mathrm{H}$, $\left.\left(\mathrm{CH}_{3}\right)_{2}\right), 1.63-1.67\left(\mathrm{~m}, 2 \mathrm{H}, \mathrm{CCH}_{2} \mathrm{CH}_{2} \mathrm{CH}_{2}\right), 1.77-1.83\left(\mathrm{~m}, 2 \mathrm{H}, \mathrm{CCH}_{2} \mathrm{CH}_{2} \mathrm{CH}_{2}\right), 2.36$ (d, J = 0.9 $\left.\mathrm{Hz}, 3 \mathrm{H}, \mathrm{CH}_{3}\right), 2.76\left(\mathrm{t}, J=6.3 \mathrm{~Hz}, 1 \mathrm{H}, \mathrm{CCH}_{2} \mathrm{CH}_{2} \mathrm{CH}_{2}\right), 3.52\left(\mathrm{~s}, 3 \mathrm{H}, \mathrm{OCH}_{3}\right), 7.16(\mathrm{~d}, J=8.4 \mathrm{~Hz}, 1 \mathrm{H}$, aromatic), 7.23 (d, $J=8.4 \mathrm{~Hz}, 1 \mathrm{H}$, aromatic), 7.29 (d, $J=1.5 \mathrm{~Hz}, 1 \mathrm{H}, \mathrm{OCH})$; MS: $\mathrm{m} / z .315$ (M $+\mathrm{H}^{+}$). Methyl 2-(1-methoxy-5,5-dimethyl-5,6,7,8-tetrahydronaphthalen-2-yl)-4-methylfuran-3carboxylate (22): ${ }^{1} \mathrm{H}$ NMR $\left(300 \mathrm{MHz}, \mathrm{CDCl}_{3}, \mathrm{ppm}\right): \delta 1.30\left(\mathrm{~s}, 6 \mathrm{H},\left(\mathrm{CH}_{3}\right)_{2}\right), 1.63-1.67(\mathrm{~m}, 2 \mathrm{H}$, $\mathrm{CCH}_{2} \mathrm{CH}_{2} \mathrm{CH}_{2}$ ), 1.77-1.83 (m, 2H, $\left.\mathrm{CCH}_{2} \mathrm{CH}_{2} \mathrm{CH}_{2}\right), 2.20$ (d, J=1.2 Hz, 3H, CH $\mathrm{CH}_{3}, 2.75$ (t, J=6.3 $\left.\mathrm{Hz}, 1 \mathrm{H}, \mathrm{CCH}_{2} \mathrm{CH}_{2} \mathrm{CH}_{2}\right), 3.46\left(\mathrm{~s}, 3 \mathrm{H}, \mathrm{OCH}_{3}\right), 3.72\left(\mathrm{~s}, 3 \mathrm{H}, \mathrm{COOCH}_{3}\right), 7.14(\mathrm{~d}, J=8.1 \mathrm{~Hz}, 1 \mathrm{H}$, aromatic), 7.23 (d, $J=8.1 \mathrm{~Hz}, 1 \mathrm{H}$, aromatic), 7.27 (d, $J=0.9 \mathrm{~Hz}, 1 \mathrm{H}, \mathrm{OCH})$; MS: $m / z, 329\left(\mathrm{M}+\mathrm{H}^{+}\right)$. 2-(3-(Hydroxymethyl)-4-methylfuran-2-yl)-5,5-dimethyl-5,6,7,8-tetrahydronaphthalene-1-ol (23): ${ }^{1} \mathrm{H}$ NMR (300 MHz, $\left.\mathrm{CDCl}_{3}, \mathrm{ppm}\right): \delta 1.30\left(\mathrm{~s}, 6 \mathrm{H},\left(\mathrm{CH}_{3}\right)_{2}\right), 1.63-1.67(\mathrm{~m}, 2 \mathrm{H}$, $\mathrm{CCH}_{2} \mathrm{CH}_{2} \mathrm{CH}_{2}$ ), 1.80-1.84 (m, $2 \mathrm{H}, \mathrm{CCH}_{2} \mathrm{CH}_{2} \mathrm{CH}_{2}$ ), 2.11 (d, J=0.9 Hz, 3H, CH 3 ), 2.71 (t, $J=6.3$ $\mathrm{Hz}, 2 \mathrm{H}, \mathrm{CCH}_{2} \mathrm{CH}_{2} \mathrm{CH}_{2}$ ), $4.58\left(\mathrm{~s}, 1 \mathrm{H}, \mathrm{CH}_{2} \mathrm{OH}\right), 6.97$ (d, $J=8.4 \mathrm{~Hz}, 1 \mathrm{H}$, aromatic), 7.20 (d, $J=8.4$ $\mathrm{Hz}, 1 \mathrm{H}$, aromatic), $7.28(\mathrm{~d}, J=0.9 \mathrm{~Hz}, 1 \mathrm{H}, \mathrm{OCH})$; MS: $m / z 385\left(\mathrm{M}-\mathrm{H}^{+}\right)$. (2-(1-methoxy-5,5dimethyl-5,6,7,8-tetrahydronaphthalen-2-yl)-4-methylfuran-3-yl)methanol (24): ${ }^{1} \mathrm{H}$ NMR (300 $\left.\mathrm{MHz}, \mathrm{CDCl}_{3}, \mathrm{ppm}\right): \delta 1.30\left(\mathrm{~s}, 6 \mathrm{H},\left(\mathrm{CH}_{3}\right)_{2}\right), 1.64-1.68\left(\mathrm{~m}, 2 \mathrm{H}, \mathrm{CCH}_{2} \mathrm{CH}_{2} \mathrm{CH}_{2}\right), 1.77-1.83(\mathrm{~m}, 2 \mathrm{H}$, $\left.\mathrm{CCH}_{2} \mathrm{CH}_{2} \mathrm{CH}_{2}\right), 2.12\left(\mathrm{~d}, J=0.9 \mathrm{~Hz}, 3 \mathrm{H}, \mathrm{CH}_{3}\right), 2.69\left(\mathrm{t}, J=6.3 \mathrm{~Hz}, 1 \mathrm{H}, \mathrm{CH}_{2} \mathrm{OH}\right), 2.77(\mathrm{t}, J=6.3$ $\left.\mathrm{Hz}, 2 \mathrm{H}, \mathrm{CCH}_{2} \mathrm{CH}_{2} \mathrm{CH}_{2}\right), 3.46\left(\mathrm{~s}, 3 \mathrm{H}, \mathrm{OCH}_{3}\right), 4.41\left(\mathrm{~d}, \mathrm{~J}=5.7 \mathrm{~Hz}, 2 \mathrm{H}, \mathrm{CH}_{2} \mathrm{OH}\right), 7.16-7.22(\mathrm{~m}, 2 \mathrm{H}$, aromatic), $7.27(\mathrm{~d}, J=0.9 \mathrm{~Hz}, 1 \mathrm{H}, \mathrm{OCH})$; MS: $m / z 323\left(\mathrm{M}+\mathrm{Na}^{+}\right)$. (2-(1-Ethoxy-5,5-dimethyl-5,6,7,8tetrahydronaphthalen-2-yl)-4-methylfuran-(2-(1-methoxy-5,5-dimethyl-5,6,7,8tetrahydronaphthalen-2-yl)-4-methylfuran-3-yl)methanol (24): ${ }^{1} \mathrm{H} \mathrm{NMR}\left(300 \mathrm{MHz}, \mathrm{CDCl}_{3}\right.$, ppm): $\delta 1.30\left(\mathrm{~s}, 6 \mathrm{H},\left(\mathrm{CH}_{3}\right)_{2}\right), 1.64-1.68\left(\mathrm{~m}, 2 \mathrm{H}, \mathrm{CCH}_{2} \mathrm{CH}_{2} \mathrm{CH}_{2}\right), 1.77-1.83\left(\mathrm{~m}, 2 \mathrm{H}, \mathrm{CCH}_{2} \mathrm{CH}_{2} \mathrm{CH}_{2}\right), 2.12$ $\left(\mathrm{d}, J=0.9 \mathrm{~Hz}, 3 \mathrm{H}, \mathrm{CH}_{3}\right), 2.69\left(\mathrm{t}, J=6.3 \mathrm{~Hz}, 1 \mathrm{H}, \mathrm{CH}_{2} \mathrm{OH}\right), 2.77(\mathrm{t}, J=6.3 \mathrm{~Hz}, 2 \mathrm{H}$,

$\mathrm{CCH}_{2} \mathrm{CH}_{2} \mathrm{CH}_{2}$ ), 3.46 (s, 3H, $\mathrm{OCH}_{3}$ ), 4.41 (d, $J=5.7 \mathrm{~Hz}, 2 \mathrm{H}, \mathrm{CH}_{2} \mathrm{OH}$ ), 7.16-7.22 (m, 2H, aromatic), $7.27(\mathrm{~d}, J=0.9 \mathrm{~Hz}, 1 \mathrm{H}, \mathrm{OCH})$; MS: $m / z 323\left(\mathrm{M}+\mathrm{Na}^{+}\right)$. (2-(1-Ethoxy-5,5-dimethyl-5,6,7,8-

tetrahydronaphthalen-2-yl)-4-methylfuran-3-yl)methanol (25): ${ }^{1} \mathrm{H}$ NMR $\left(300 \mathrm{MHz}, \mathrm{CDCl}_{3}, \mathrm{ppm}\right)$ : $\delta 1.18\left(\mathrm{t}, J=7.2 \mathrm{~Hz}, 3 \mathrm{H}, \mathrm{CH}_{2} \mathrm{CH}_{3}\right), 1.30\left(\mathrm{~s}, 6 \mathrm{H},\left(\mathrm{CH}_{3}\right)_{2}\right), 1.64-1.68\left(\mathrm{~m}, 2 \mathrm{H}, \mathrm{CCH}_{2} \mathrm{CH}_{2} \mathrm{CH}_{2}\right), 1.77-$ $1.83\left(\mathrm{~m}, 2 \mathrm{H}, \mathrm{CCH}_{2} \mathrm{CH}_{2} \mathrm{CH}_{2}\right), 2.12\left(\mathrm{~d}, J=0.9 \mathrm{~Hz}, 3 \mathrm{H}, \mathrm{CH}_{3}\right), 2.76(\mathrm{t}, J=6.3 \mathrm{~Hz}, 1 \mathrm{H}$, $\mathrm{CCH}_{2} \mathrm{CH}_{2} \mathrm{CH}_{2}$ ), 2.87 (br, $\left.1 \mathrm{H}, \mathrm{CH}_{2} \mathrm{OH}\right), 3.58\left(\mathrm{q}, \mathrm{J}=7.2 \mathrm{~Hz}, 2 \mathrm{H}, \mathrm{CH}_{2} \mathrm{CH}_{3}\right), 4.39$ (s, $\left.2 \mathrm{H}, \mathrm{CH}_{2} \mathrm{OH}\right)$, 7.18 (s, 2H, aromatic), $7.26(\mathrm{~d}, J=0.3 \mathrm{~Hz}, 1 \mathrm{H}, \mathrm{OCH})$; MS: $\mathrm{m} / z .313\left(\mathrm{M}-\mathrm{H}^{+}\right) .2-(1-$ Methoxy-5,5dimethyl-5,6,7,8-tetrahydronaphthalen-2-yl)-3-(methoxymethyl)-4-me thylfuran (26). $44 \%$ yield; ${ }^{1} \mathrm{H}$ NMR $\left(300 \mathrm{MHz}, \mathrm{CDCl}_{3}\right.$, ppm): $\delta 1.30\left(\mathrm{~s}, 6 \mathrm{H},\left(\mathrm{CH}_{3}\right)_{2}\right), 1.64-1.67(\mathrm{~m}, 2 \mathrm{H}$, $\left.\mathrm{CCH}_{2} \mathrm{CH}_{2} \mathrm{CH}_{2}\right), 1.77-1.83\left(\mathrm{~m}, 2 \mathrm{H}, \mathrm{CCH}_{2} \mathrm{CH}_{2} \mathrm{CH}_{2}\right), 2.10\left(\mathrm{~d}, J=1.2 \mathrm{~Hz}, 3 \mathrm{H}, \mathrm{CH}_{3}\right), 2.77$ (t, $J=6.3$ $\left.\mathrm{Hz}, 1 \mathrm{H}, \mathrm{CCH}_{2} \mathrm{CH}_{2} \mathrm{CH}_{2}\right), 3.33\left(\mathrm{~s}, 3 \mathrm{H}, \mathrm{CH}_{2} \mathrm{OCH}_{3}\right), 3.49$ (s, $\left.3 \mathrm{H}, \mathrm{OCH}_{3}\right), 4.32\left(\mathrm{~s}, 2 \mathrm{H}, \mathrm{CH}_{2} \mathrm{OCH}_{3}\right)$, 
7.17 (dd, $J=8.4 \mathrm{~Hz}, 2 \mathrm{H}$, aromatic), $7.28(\mathrm{~d}, J=1.2 \mathrm{~Hz}, 1 \mathrm{H}, \mathrm{OCH})$; MS: $m / z 315\left(\mathrm{M}+\mathrm{H}^{+}\right) .2-(1-$ Ethoxy-5,5-dimethyl-5,6,7,8-tetrahydronaphthalen-2-yl)-3-(methoxymethyl)-4-methylfuran (27): ${ }^{1} \mathrm{H}$ NMR (300 MHz, $\left.\mathrm{CDCl}_{3}, \mathrm{ppm}\right): \delta 1.19\left(\mathrm{t}, J=7.2 \mathrm{~Hz}, 3 \mathrm{H}, \mathrm{CH}_{2} \mathrm{CH}_{3}\right), 1.30\left(\mathrm{~s}, 6 \mathrm{H},\left(\mathrm{CH}_{3}\right)_{2}\right)$, 1.64-1.67 (m, 2H, $\left.\mathrm{CCH}_{2} \mathrm{CH}_{2} \mathrm{CH}_{2}\right), 1.77-1.83\left(\mathrm{~m}, 2 \mathrm{H}, \mathrm{CCH}_{2} \mathrm{CH}_{2} \mathrm{CH}_{2}\right), 2.09$ (d, J = 0.9 Hz, 3H, $\left.\mathrm{CH}_{3}\right), 2.77\left(\mathrm{t}, J=6.3 \mathrm{~Hz}, 1 \mathrm{H}, \mathrm{CCH}_{2} \mathrm{CH}_{2} \mathrm{CH}_{2}\right), 3.32\left(\mathrm{~s}, 3 \mathrm{H}, \mathrm{CH}_{2} \mathrm{OCH}_{3}\right), 3.59(\mathrm{q}, J=6.9 \mathrm{~Hz}, 2 \mathrm{H}$, $\left.\mathrm{CH}_{2} \mathrm{CH}_{3}\right), 4.32\left(\mathrm{~s}, 2 \mathrm{H}, \mathrm{CH}_{2} \mathrm{OCH}_{3}\right), 7.16(\mathrm{dd}, J=8.1 \mathrm{~Hz}, 2 \mathrm{H}$, aromatic), $7.26(\mathrm{~d}, J=0.9 \mathrm{~Hz}, 1 \mathrm{H}$, $\mathrm{OCH}$ ); MS: $\mathrm{m} / z, 329\left(\mathrm{M}+\mathrm{H}^{+}\right)$. (2-(1-Methoxy-5,5-dimethyl-5,6,7,8-tetrahydronaphthalen-2-yl)-4methylfuran-3-yl)methyl acetate (28): ${ }^{1} \mathrm{H}$ NMR (300 MHz, $\left.\mathrm{CDCl}_{3}, \mathrm{ppm}\right): \delta 1.30\left(\mathrm{~s}, 6 \mathrm{H},\left(\mathrm{CH}_{3}\right)_{2}\right)$, 1.63-1.67 (m, 2H, $\left.\mathrm{CCH}_{2} \mathrm{CH}_{2} \mathrm{CH}_{2}\right), 1.77-1.83\left(\mathrm{~m}, 2 \mathrm{H}, \mathrm{CCH}_{2} \mathrm{CH}_{2} \mathrm{CH}_{2}\right), 2.06(\mathrm{~s}, 3 \mathrm{H}$,

$\left.\mathrm{CH}_{2} \mathrm{OCOCH}_{3}\right), 2.06\left(\mathrm{~d}, J=0.9 \mathrm{~Hz}, 3 \mathrm{H}, \mathrm{CH}_{3}\right), 2.76\left(\mathrm{t}, \mathrm{J}=6.3 \mathrm{~Hz}, 1 \mathrm{H}, \mathrm{CCH}_{2} \mathrm{CH}_{2} \mathrm{CH}_{2}\right), 3.48(\mathrm{~s}, 3 \mathrm{H}$, $\left.\mathrm{OCH}_{3}\right), 5.01\left(\mathrm{~s}, 2 \mathrm{H}, \mathrm{CH}_{2} \mathrm{OCOCH}_{3}\right), 7.13-7.19$ (m, 2H, aromatic), 7.30 (d, J=1.2 Hz, 1H, OCH); MS: $m / z, 365\left(\mathrm{M}+\mathrm{Na}^{+}\right)$. 
<smiles></smiles>

Tanshinone I (1)<smiles>Cc1coc2c1ccc1c3cccc(C)c3c(=O)oc12</smiles>

Neo-tanshinlatone I (3)<smiles>[R]Cc1ccc2c(oc(=O)c3c(C)coc32)c1N(C)CCC</smiles>

5<smiles>Cc1coc2c1C(=O)C(=O)c1c-2ccc2c1CCCC2(C)C</smiles>

Tanshinone IIA (2)<smiles>CCc1cccc2c(O)c(-c3occ(C)c3C(=O)O)ccc12</smiles>

4<smiles>[R]Cc1ccc(-c2occ(C)c2C(=O)O)c(O)c1NCCCC</smiles>

6

Figure 1.

Structures of tanshinone I (1), tanshinone IIA (2), neo-tanshinlactone (3), analog 4, and two newly designed scaffolds 5-6 
<smiles>COc1ccccc1CCC=C(C)C</smiles>

7

8

9

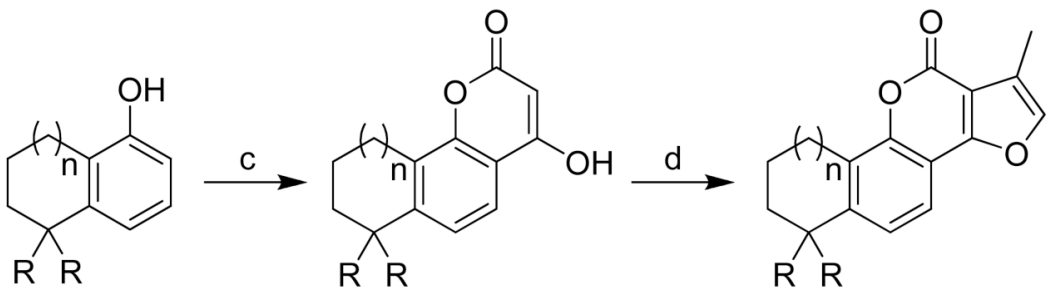

10, $\mathrm{n}=0, \mathrm{R}=\mathrm{H}$

11, $n=1, R=H$

12, $\mathrm{n}=0, \mathrm{R}=\mathrm{H}$

13, $\mathrm{n}=1, \mathrm{R}=\mathrm{H}$

9, $n=1, R=M e$

14, $n=1, R=M e$

15, $\mathrm{n}=0, \mathrm{R}=\mathrm{H}$

16, $n=1, R=H$

17, $n=1, R=M e$

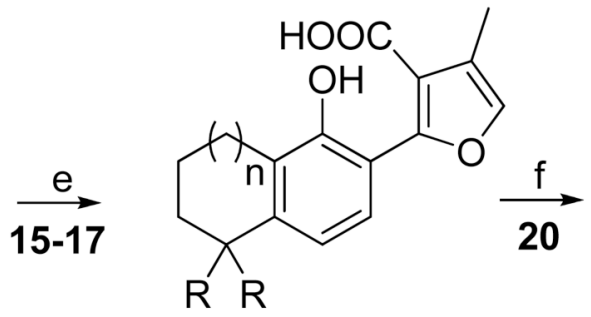

18, $n=0, R=H$

$19, \mathrm{n}=1, \mathrm{R}=\mathrm{H}$

20, $n=1, R=M e$<smiles>COC(=O)c1c(C)coc1-c1ccc2c(c1O)CCCC2(C)C</smiles>

21<smiles>COC(=O)c1c(C)coc1-c1ccc2c(c1OC)CCC(C)(C)C2(C)C</smiles>

22<smiles>Cc1coc(-c2ccc3c(c2O)CCCC3(C)C)c1CO</smiles>

23<smiles>[R]Oc1c(-c2occ(C)c2CO)ccc2c1CCCC2(C)C</smiles>

24: $\mathrm{R}=\mathrm{Me}$ 25: $\mathrm{R}=\mathrm{Et}$<smiles>[R]OCc1c(C)coc1-c1ccc2c(c1OC)CCCC2(C)C</smiles>

26: $\mathrm{R}=\mathrm{Me}$

27: $R=E t$

Scheme 1.

Reactions and conditions: (a) 4-methylpent-3-enylzinc(II) bromide, $\mathrm{Pd}\left(\mathrm{Cl}_{2}\right)$ (dppf), THF, reflux, 1h; (b) (i) $\mathrm{AlCl}_{3}, \mathrm{DCM}, 0{ }^{\circ} \mathrm{C}, 15 \mathrm{~min}$; (ii) $\mathrm{BBr}_{3}, \mathrm{CH}_{2} \mathrm{Cl}_{2}$; (c) malonic acid, $\mathrm{PPA}, 75^{\circ}$ $\mathrm{C}, 3 \mathrm{~h}$; (d) chloroacetone, $\mathrm{HOAc} / \mathrm{NH}_{4} \mathrm{OAc}$, toluene/EtOH, reflux, $24 \mathrm{~h}$; (e) $5 \% \mathrm{NaOH}$ (aq), reflux; (f) $\mathrm{NaOH}, 18$-crown-6, MeI, $\mathrm{CH}_{3} \mathrm{CN}, 90{ }^{\circ} \mathrm{C}$; (g) $\mathrm{SOCl}_{2}, \mathrm{MeOH}$, rt; (h) $\mathrm{LiAlH}_{4}$, THF; (i) $\mathrm{MeI}$ or EtI, $\mathrm{Cs}_{2} \mathrm{CO}_{3}$, acetone, $50{ }^{\circ} \mathrm{C}$; (g) MeI or EtI, NaH, THF, rt; (k) $\mathrm{Ac}_{2} \mathrm{O}, \mathrm{Et}_{3} \mathrm{~N}, \mathrm{DMAP}$, $\mathrm{CH}_{2} \mathrm{Cl}_{2}$. 


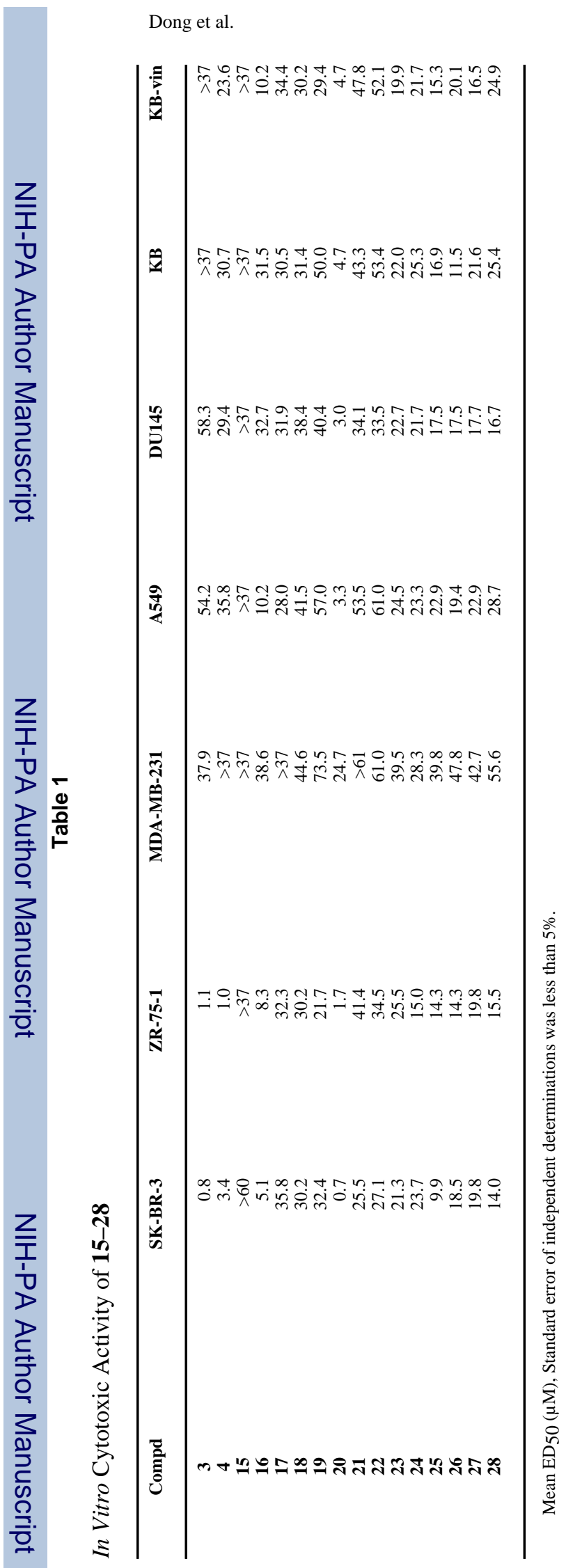

Page 8

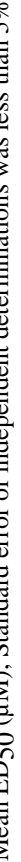

Bioorg Med Chem Lett. Author manuscript; available in PMC 2010 November 15. 